Щ्)FRANÇAISE

$\supset$ DE

뙴 PÉDAGOGIE

\section{Revue française de pédagogie}

Recherches en éducation

$198 \mid 2017$

Regards croisés sur le baccalauréat professionnel

\title{
GARNIER Pascale, BROUGÈRE Gilles, RAYNA Sylvie \& RUPIN Pablo. À 2 ans, vivre dans un collectif d'enfants. Crèche, école maternelle, classe passerelle, jardin maternel
}

Toulouse : Érès, 2016, 360 p.

Éric Plaisance

\section{(2) OpenEdition \\ Journals}

\section{Édition électronique}

URL : https://journals.openedition.org/rfp/5391

DOI : 10.4000/rfp.5391

ISSN : 2105-2913

Éditeur

ENS Éditions

Édition imprimée

Date de publication : 31 mars 2017

Pagination : 115-119

ISSN : 0556-7807

Référence électronique

Éric Plaisance, «GARNIER Pascale, BROUGĖRE Gilles, RAYNA Sylvie \& RUPIN Pablo. À 2 ans, vivre dans un collectif d'enfants. Crèche, école maternelle, classe passerelle, jardin maternel », Revue française de pédagogie [En ligne], 198| 2017, mis en ligne le 31 mars 2017, consulté le 08 janvier 2022. URL : http:// journals.openedition.org/rfp/5391; DOI : https://doi.org/10.4000/rfp.5391

Ce document a été généré automatiquement le 8 janvier 2022

(c) tous droits réservés 


\section{GARNIER Pascale, BROUGÈRE Gilles, RAYNA Sylvie \& RUPIN Pablo. À 2 ans, vivre dans un collectif d'enfants. Crèche, école maternelle, classe passerelle, jardin maternel}

Toulouse : Érès, 2016, 360 p.

Éric Plaisance

\section{RÉFÉRENCE}

GARNIER Pascale, BROUGÈRE Gilles, RAYNA Sylvie \& RUPIN Pablo. À 2 ans, vivre dans un collectif d'enfants. Crèche, école maternelle, classe passerelle, jardin maternel. Toulouse : Érès, 2016, $360 \mathrm{p}$.

Les publications sur les structures d'accueil et d'éducation de la petite enfance se sont le plus souvent focalisées sur tel ou tel type d'institutions, reprenant ainsi le découpage officiel français entre celles qui relèvent de la tutelle du ministère de l'Éducation nationale (les écoles maternelles) et celles qui relèvent de la tutelle du ministère des Affaires sociales (dont le nom même a souvent changé au cours de l'histoire récente, par exemple ministère de la Famille et des Affaires sociales, etc.). Un découpage institutionnel qui pourrait passer pour évident puisqu'il semble logiquement reposer sur le découpage des âges des enfants concernés, les plus jeunes se situant dans les structures des Affaires sociales, les plus âgés dans les structures de l'Éducation nationale. Mais c'est une évidence en trompe l'œil. D'abord parce que d'autres pays ne se situent nullement dans cet arbitraire institutionnel qui provient de notre histoire (et qui perdure dans une sorte d'enkystement à la fois administratif et mental), et ont, au contraire, unifié les tutelles sous l'égide de l'Éducation nationale pour l'ensemble des 
âges de 0 à 6 ans, comme les pays scandinaves. Ensuite, parce qu'en France même, la superposition des compétences institutionnelles pour les enfants âgés de 2 à 3 ans (qui peuvent encore rester en crèche ou aller déjà en école maternelle) ne laisse pas de susciter des débats sans fin, voire des polémiques, ou des propos caricaturaux, par exemple chez certains hommes politiques prenant position sur la formation jugée trop longue des professeurs d'école maternelle s'occupant des enfants les plus jeunes! Paradoxalement, peu de chercheurs ont porté leur regard sur ces âges charnières et sur les éventuelles différences de comportement des enfants de ces mêmes âges selon les environnements qui les accueillent, leurs modes de fonctionnement, les normes éducatives plus ou moins explicitées et les professionnelles impliquées. C'est pourtant dans un livre ancien et un peu oublié que Bianka Zazzo (1984) avait déjà posé la question encore présente aujourd'hui «l'école maternelle à 2 ans : oui ou non?", mais en esquissant des éléments de réponses nuancées à partir de la mise en place d'expérimentations ingénieuses. Avec des installations matérielles, soit proches d'une crèche, soit proches d'une classe maternelle, elle montrait des espaces différemment structurés qui influençaient les comportements des enfants. C'est dire qu'elle ne répondait pas abruptement à la question du titre même de son livre mais insistait sur le rôle essentiel de la qualité générale des conditions d'accueil et, pour la maternelle, sur la nécessité d'une mobilisation prioritaire des adultes pour la fréquentation des plus jeunes.

2 Ce bref rappel d'éléments de contexte permet de montrer la grande originalité de cet ouvrage collectif à quatre voix qui propose un regard transversal sur les différentes structures pour les enfants de 2 ans, plus exactement sur l'accueil en crèche, en école maternelle et dans deux autres structures "intermédiaires", plus rares et moins connues, la classe passerelle et le jardin maternel. En début d'ouvrage, les auteurs prennent bien soin de présenter ces structures dans leur cadrage institutionnel où se jouent des tutelles et des initiatives différentes, par exemple de la part de municipalités ou d'associations. Leur objectif est précisément celui-ci : «investir une pluralité des modes d'accueil et d'éducation des enfants de cette tranche d'âge» et, dans ces conditions, se donner « les moyens de dessiner un continuum de structures qui dépasse le clivage entre école maternelle et crèche » (p.11). Ou encore : " montrer comment se joue la vie en collectivité d'enfants de 2-3 ans dans quatre structures différentes " (p. 21). Ceci sans prétendre à une représentation statistique des terrains enquêtés.

3 Encore faut-il que les auteurs s'engagent dans des questionnements précis qui donnent sens et consistance aux descriptions des réalités vécues par les enfants et les professionnelles. Et c'est là que réside l'autre originalité de l'ouvrage, sur le plan des concepts utilisés et des méthodologies mises en œuvre. Leur parti-pris est de se tenir à distance d'une illusoire neutralité surplombante d'observateurs, car tout savoir, y compris celui des chercheurs, est toujours situé, tributaire des contextes et des points de vue adoptés. Pour autant, il faut savoir se rapprocher des expériences vécues par les enfants et par les professionnelles, savoir adopter des points de vue « d'en bas » (selon l'expression citée de Haraway), en puisant dans des méthodologies variées: observations de terrain, entretiens, vidéos, photos, etc., c'est-à-dire avec diverses approches qualitatives où le point de vue des enfants est posé en toute priorité. Et là encore, l'objectif n'est pas de mettre en parallèle des pratiques et des normes de fonctionnement en essayant de définir ce qui est meilleur pour les enfants (avec cette fiction ravageuse des «bonnes pratiques"!), mais de recueillir, à partir de situations 
singulières, les différentes modalités de socialisation où les enfants sont en première ligne. Un exemple frappant de cette option méthodologique est l'utilisation par les enfants eux-mêmes d'appareils photo grâce auxquels ils peuvent photographier ce qui leur semble important dans leur environnement. Un ensemble de photos prises par les enfants est présenté dans plusieurs encarts couleur au centre de l'ouvrage et on y voit aussi bien des installations de jeux, d'autres enfants, une plaque d'égout qu'un vase sanitaire de WC! On repère ici ce souci de situer l'enfant dans ses propres intérêts, de le poser comme acteur social, selon l'orientation fondamentale de la sociologie de l'enfance, pourtant peu évoquée comme telle dans le texte des auteurs. Ce privilège revendiqué aux approches visuelles est aussi bien illustré par les montages audiovisuels de situations qui servent de supports aux échanges avec les parents et avec les professionnelles. Dans ce dernier cas, il s'agit de «susciter une prise de distance à l'égard de [ses] propres pratiques, de faire émerger des débats, des valeurs, des représentations » (p. 18).

4 Mais c'est un ensemble de concepts qui permet de structurer les observations et de proposer régulièrement des interprétations. Ils sont présentés en début d'ouvrage, ce qui permet au lecteur d'avoir une sorte de guide pour se repérer parmi les très nombreux extraits d'observations et de dialogues entre enfants et entre adultes et enfants. Présentation indispensable car les auteurs empruntent le plus souvent à la langue anglaise les concepts qui leur semblent les plus importants.

Ainsi, les concepts de :

- social setting, qui « représente les règles qui régissent le temps et l'espace, et plus encore leur relation» (p.13), ou encore « comme une structure spatio-temporelle qui met constamment en relation les usages légitimes de l'espace avec le temps » (p. 30). Ainsi, les enfants repèrent bien, dans les lieux où ils sont présents, ce qu'il convient de faire « au bon moment » et « au bon endroit »;

- script, souvent dénommé script institutionnel, comme un ensemble d'invitations à agir, le plus souvent en relation avec des objets. Ainsi : «On peut considérer que le script qui accompagne l'objet, telle une poupée, invite à des actions qui constituent le répertoire des performances associées à l'objet » (p. 14);

- répertoire de pratiques, expression utilisée cette fois-ci en français mais en réalité inspirée d'auteurs anglophones (comme Barbara Rogoff et collaborateurs, in Brougère \& Vandenbroeck, 2007) et qui renvoie, dans les interactions quotidiennes, à la fois aux capacités d'action des individus (aussi bien des enfants que des professionnelles) et au cadre culturel dans lequel ils sont inscrits. Dans ces conditions, «à travers le script institutionnel, les performances des professionnelles, les performances des enfants validées par celles-ci, se met en place un répertoire de pratiques légitimes propres à chaque structure » (p. 15);

- affordance, notion non mentionnée par les auteurs en début de texte mais utilisée à plusieurs reprises, comme ce qu'offre une structure ou un objet pour le développement d'activités, pour diverses explorations, par exemple grâce à l'appareil photo aux mains des enfants.

6 C'est donc munis de ces cadres théoriques que les auteurs rendent compte de leurs observations de la diversité des structures enquêtées et des modes de comportement des enfants et des adultes. Ce qui apparaît nettement, c'est, bien entendu, la relativité institutionnelle, depuis la crèche qui accompagne les enfants plus qu'elle ne les oriente, jusqu'à la classe de maternelle où le cadrage est nettement plus présent. Mais ce qui 
frappe le lecteur, c'est la capacité extraordinaire des enfants du même âge de savoir se situer dans des cadres de vie différents et même de savoir jouer avec les normes des adultes, de se montrer ainsi comme des acteurs sociaux. De beaux exemples sont fournis à propos de la classe des tout-petits en maternelle. À la cantine, pendant le temps long du repas, certains enfants sortent de l'interdit de jouer avec la nourriture et se mettent du pain sur la tête, ou encore mettent leur nez dans les verres. Selon les observateurs, « le repas apparaît comme une performance qui s'éloigne très souvent du script et permet initiative et détournements malgré les rappels à l'ordre répétés » (p.75). Plus généralement, les relations réciproques entre les enfants paraissent s'installer dans ce que les auteurs appellent joliment les « interstices» de la structure et des activités permises. Lorsque les enfants, toujours les tout-petits en maternelle, participent à la visite d'un aquarium où se trouvent des requins, ils interagissent bruyamment en ayant un peu peur, différencient ensemble les requins "papa " ou «maman», et sont donc capables de produire une action collective. Mais pour les chercheurs, ce qui semble dominer dans cette petite section, c'est le rôle central des adultes qui organisent les situations et contrôlent le cadre spatio-temporel. De courts éléments de comparaison avec l'Allemagne montrent un fort contraste avec les jardins d'enfants de ce pays où n'apparaît pas la soumission à un rythme collectif et où la socialisation des jeunes enfants va de pair avec l'individualisation des activités

7 Les situations dans cette classe maternelle sont minutieusement décrites et sont l'occasion de reprendre systématiquement le concept de « répertoire de pratiques » en en distinguant plusieurs modalités (p.98):1) les pratiques légitimes, conformes à ce qui est attendu d'une classe maternelle où l'enseignante délivre le sens d'une activité, voire demande l'application d'une consigne. Pour autant, les enfants participent volontiers, certes à des degrés variables, mais en adhérant aux routines dictées par les adultes. C'est ici que l'on pourrait nuancer les propos des auteurs en rappelant à quel point les jeunes enfants aiment la répétition d'activités, la lecture de la même histoire dans les mêmes termes, comme si la routine leur donnait un sentiment de permanence rassurante et une prise sur les réalités ; 2) les pratiques qui sortent du cadre légitime, "parfois tolérées, souvent interdites", qui concernent principalement le corps : danse, interactions dans le jeu, explorations, et où se déploient des interactions entre enfants, par exemple aux moments d'attente en fin de journée ; 3) les pratiques ouvertes, à michemin entre les deux premiers types, par exemple dans les récréations ou les activités en coins jeux. Ici les observateurs repèrent un répertoire de cultures ludiques mais diversement mises en application en fonction du cadre spatio-temporel et des échappatoires possibles.

On comprend alors le contraste entre les activités de la classe maternelle et celles du jardin maternel enquêté. $\mathrm{Si}$, dans le premier cas, les observateurs perçoivent déjà des "rails scolaires" (p. 331) et des attentes orientées vers un "métier d'élève », selon l'expression reprise à Philippe Perrenoud, dans la deuxième situation, celle du jardin maternel, ce sont les interactions qui retiennent l'attention, celles entre les enfants et les adultes et celles entre les enfants eux-mêmes. Donc double dimension des relations, double dimension de la socialisation, verticale d'un côté, horizontale de l'autre. Ce qui fait penser à un auteur non mentionné, Ana Vasquez, qui avait précisément travaillé sur ces aspects à propos de l'école primaire (Vasquez-Bronfman \& Martinez, 1996). Ana Vasquez avait pu montrer que, dans les relations horizontales entre enfants, une certaine clandestinité se faisait jour, en marge des adultes, mais fondamentale dans la vie quotidienne des enfants. Dans ce jardin maternel, qui apparaît pour les enfants 
comme la première prise en charge collective hors de la famille, le trait essentiel est la souplesse des activités proposées aux enfants à travers une culture matérielle, c'est-àdire par l'intermédiaire d'objets divers qui balisent leur parcours quotidien. L'attention " micro " accordée à un ou deux enfants précis montre l'importance des moments «non cadrés» et des pratiques «ouvertes»(p. 247). C'est, par exemple, la libre circulation des enfants, qui leur permet les changements de lieux, le détournement possible de l'usage des objets grâce à leur inventivité (l'usage de pseudo microphones, la transformation de la pâte à sel en gâteau...), la facilitation des interactions et des jeux symboliques entre eux (par exemple avec des objets qui représentent de la nourriture), en bref, des moments d'apprentissage informel. D'autres moments plus encadrés par les adultes fonctionnent néanmoins, comme ceux des repas où la manipulation des couverts est contrôlée, ou encore ceux de la lecture collective, où l'immobilité relative du corps est requise. Mais sur l'ensemble des observations produites, c'est l'importance accordée au jeu, à la liberté de mouvement et aux interactions qui suggère l'expression de "métier d'enfant» (p. 253), en contraste avec celle de "métier d'élève », utilisée précédemment. Rappelons que ce "métier d'enfant» est la belle expression qui a été utilisée par la célèbre inspectrice générale des écoles maternelles, Pauline Kergomard, pour défendre précisément la place du jeu en école maternelle. Sa formule était la suivante en 1886: «Le jeu c'est le travail de l'enfant; c'est son métier, c'est sa vie. L'enfant qui joue à l'école maternelle s'initie à la vie sociale, et l'on oserait dire qu'il n'apprend rien en jouant?»

9 L'autre structure, la classe passerelle, est, par définition, un " entre-deux » : pas encore une école, mais déjà autre que la crèche, permettant "un tissage entre différents répertoires de pratiques qui empruntent à ces deux mondes» (p. 106). Ce type de structure a pu être créé à partir de 1990 grâce à un partenariat entre l'Éducation nationale et le secrétariat de la Famille et des Affaires sociales. L'objectif était d'accueillir des enfants de 2 à 3 ans issus de milieux défavorisés pour leur offrir une transition entre la famille et l'école maternelle. Dans le cas présent, l'implication de la municipalité a facilité l'implantation de cette classe passerelle dans une petite école maternelle. Plusieurs traits spécifiques méritent d'être retenus : la collaboration entre professionnelles de statuts différents (professeure des écoles, éducatrice de jeunes enfants, agent territorial spécialisé) et l'accueil des parents. On retient des observations de terrain toujours très minutieusement restituées que cette structure hybride remplit pratiquement l'objectif d'établir une passerelle en souplesse. Selon la typologie précédente des répertoires de pratiques, les enfants sont ici à la croisée de différents répertoires, ils y "circulent»: "ce qu'ils sont censés faire et ce qu'ils font plus ou moins (apprendre à se comporter comme élève ou membre de la classe); ce qu'ils font en dehors du script (contournements, "contre-élève") ; ce qu'ils font dans des moments ouverts, entre enfants (récréations, jeux, explorations, conflits non modérés, etc.). En ce sens, on peut comprendre la socialisation des enfants comme l'intégration de ces différents répertoires et leur adaptation aux divers contextes socio-éducatifs » (p. 179).

Quant aux grands à la crèche, ils bénéficient, dans le cas observé et contrairement à la situation de la maternelle, de longs moments d'accueil du matin et du soir par l'échelonnement des arrivées, des moments où se déploient des activités de jeux libres, des échanges avec les adultes, y compris les parents. De manière générale, c'est l'omniprésence du jeu qui est notée et la possibilité des échanges informels, ce qui n'exclut pourtant pas les regroupements et le rôle des adultes. Ainsi, la section des grands en crèche constitue un mode de fonctionnement composite. Selon le 
vocabulaire privilégié par les auteurs de l'ouvrage, le «script» est plus souple qu'en maternelle et laisse des possibilités de choix des activités ludiques mais il annonce aussi les rythmes plus collectifs de la structure scolaire.

11 En conclusion de l'ouvrage, c'est la question des identités professionnelles qui est soulignée. Les enseignantes de maternelle sont en effet dans une tension entre l'accueil spécifique des plus jeunes enfants quand il s'agit de la petite section, et la perspective scolaire pour laquelle elles ont été formées. Pour certaines d'entre elles, s'occuper des plus petits aboutirait même à une remise en cause de leur métier: "il faut désapprendre notre métier » dit l'une d'elles de manière très significative (p. 104).

Les analyses proposées dans cet ouvrage peuvent paraître bien sévères à l'égard de la maternelle qui a longtemps bénéficié d'une réputation très favorable. Mais rappelons d'abord qu'il s'agit d'observations extrêmement détaillées seulement de la petite section pour les enfants de 2 à 3 ans, complétées par des entretiens avec les professionnelles. Soulignons ensuite que ce travail est méthodologiquement construit sur la base de comparaisons entre différentes modalités d'accueil et d'éducation. Et c'est précisément en fonction des comparaisons opérées que peuvent être montrés les forts contrastes entre des structures dites parfois «de la petite enfance» et les structures scolaires qui accueillent pourtant des enfants de la même tranche d'âge. Parfois encore, mais plus succinctement, les auteurs font appel à des éléments de comparaison internationale, en citant la place occupée par le jeu en Allemagne dans les kindergarten. La productivité de ces démarches comparatives permet donc de relativiser ce qu'ils appellent les scripts institutionnels, c'est-à-dire de montrer la diversité des modes de socialisation des jeunes enfants, la diversité des normes de fonctionnement et, conjointement, les capacités enfantines à jouer avec ces normes, parfois dans les « interstices » de ce qui est défendu. En ce sens, cet ouvrage peut être considéré comme une belle contribution à une sociologie de la petite enfance qui dépasse les clivages institutionnels et pose la nécessité de mettre en chantier une réflexion d'ensemble sur les politiques d'accueil et d'éducation des enfants de moins de 6 ans.

\section{BIBLIOGRAPHIE}

BROUGÈRE G. \& VANDENBROECK M. (dir.) (2007). Repenser l'éducation des jeunes enfants. Bruxelles : P.I.E. Peter Lang.

VASQUEZ-BRONFMAN A. \& MARTINEZ I. (1996). La socialisation à l'école. Approche ethnographique. Paris : PUF.

ZAZZO B. (1984). L'école maternelle à deux ans : oui ou non ? Paris : Stock. 


\section{AUTEURS}

\section{ÉRIC PLAISANCE}

Université Paris Descartes-Sorbonne Paris Cité, Centre de recherche sur les liens sociaux 